\title{
Polarization Selective Photonic Crystal Based Integrated Plasmonic Sensor
}

\author{
Rik Chattopadhyay, Tushar Biswas, Shyamal K. Bhadra \\ CSIR-Central Glass and Ceramic Research Institute \\ 196, Raja S.C. Mullick Road, Kolkata-700032
}

\begin{abstract}
We report a two dimensional photonic crystal based integrated plasmonic sensor which selects only transverse magnetic polarization from an unpolarized light input. The device is polarization sensitive over a large band of wavelength, $590 \mathrm{~nm}$ to $833 \mathrm{~nm}$. The device is extremely compact, can be realized through batch fabrication (mass) and effective for designing on chip plasmonic sensors. The polarization selection improves the signal to noise ratio of the output signal and thus provides more detection sensitivity.
\end{abstract}

Keywords_-photonic crystal, Integrated plasmonic sensor.

\section{INTRODUCTION}

Integrated plasmonic sensors are extremely small in size, normally fabricated on planar waveguide structure and provide almost equal sensitivity like the standard Krestchmann type plasmonic sensors, which are large in size and employs bulk optics. Integrated sensors allow sensing in multiple channels simultaneously, requires minimum amount of energy for operation and their integrated design gives structural robustness. The main problem of any plasmonic sensors is that only the transverse magnetic (TM) modes excite surface plasmon. Since the modal cutoff width of the guiding layer is larger for TM mode than transverse electric (TE) mode, so it is not possible in integrated waveguide sensor to eliminate the fundamental TE mode without cutting off the fundamental TM mode. So the input light should be polarized in order to get better signal to noise ratio (SNR). Bulk plasmon sensor employs external polarizer to obtain TM polarized light which is not a good option for designing integrated sensors. Therefore a waveguide (WG) based polarizer is required for integrated sensors. The palsmonic biosensor should be sensitive around refractive index (RI) 1.33 and operable in the wavelength region $600 \mathrm{~nm}$ to $900 \mathrm{~nm}$ [1]. So far all reported waveguide based TM polarizers works on a wavelength region close to $1.55 \mu \mathrm{m}$, which is far from the requirement. We first report a two dimensional photonic crystal (PC) waveguide which allows only the TM mode in the wavelength range $590 \mathrm{~nm}$ to $833 \mathrm{~nm}$.
The PC is analyzed analytically using the plane wave expansion method (PWM) [2] and wave propagation through integrated sensor structure is done with the help of Finite Difference Time Domain (FDTD) method using Lumerical FDTD software and an estimation of the detection signal is made to predict the performance of the device. We compared the sensitivity of the proposed sensor with other reported experimental integrated sensors employing output power measurement principle.

\section{DESIGN OF INTEGRATED SENSOR}

\section{A. Design of photonic crystal}

Photonic crystal structure is an array of a high dielectric permittivity material on a low permittivity material or vice versa. The structure allows some optical frequency signal to pass through it and some frequencies are forbidden in the structure. The electromagnetic wave equation in a PC can be written as [2]

$$
\begin{aligned}
& \nabla \times[\nabla \times \boldsymbol{E}(\boldsymbol{x} \mid \omega)]=\frac{\omega^{2}}{\mathrm{c}^{2}} \varepsilon\left(\boldsymbol{x}_{\|}\right) \boldsymbol{E}(\boldsymbol{x} \mid \omega)
\end{aligned}
$$

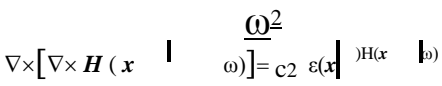

where $E(x \mid \omega)$ and $H(x \mid \omega)$ are the electric and the magnetic field of the photon, $\omega$ is the angular frequency, $\mathrm{c}$ is the velocity of light and $\varepsilon\left(\mathrm{x}_{\|}\right)$is the dielectric function describing the PC. The eigenvalue equation (1) and (2) are solved for $\omega$. Only the real values of $\omega$ are the acceptable solution. The electromagnetic (EM) wave having these frequencies can pass through the structure. The complex valued solutions form the photonic band gap (PBG) and are forbidden to pass through the PC. Therefore one can generate a plot between normalized frequency $\omega \mathrm{a} / 2 \pi \mathrm{c}$, where a is the lattice constant, and wavevector $\mathrm{k}(2 \pi / \lambda$, where $\lambda$ represents operating wavelength). The 
components of $\mathrm{k}$ in the plane of the crystal is called in-plane wave vector and the component perpendicular to the plane is called out-of-plane wave-vector. So two sets of PBG can be generated, namely in-plane PBG where normalized frequency is plotted as a function of in-plane $\mathrm{k}$ and the other is off-plane where normalized frequency is plotted against the out-of-plane $\mathrm{k}$. When a defect is introduced in the crystal then all EM waves having forbidden frequencies are confined within the defect. It is evident from Equation (1) and (2) that the band structure depends on $\varepsilon\left(\mathrm{x}_{\|}\right)$i.e. the permittivity of the materials used, lattice constants (for the proposed structure it is the distance between the centers of the silicon rods) and the dimension of the lattice sites (in the present case rod diameter). The schematic diagram of the PC structure is shown in Fig. 1.

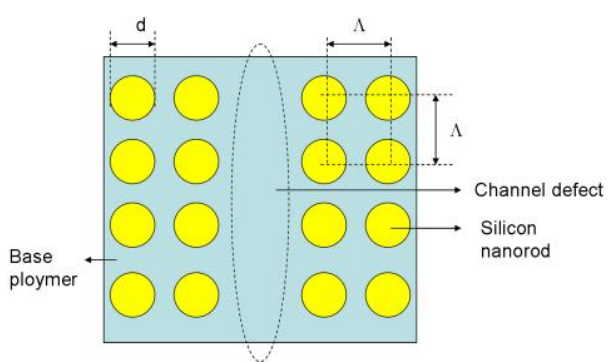

Fig. 1. Schematic diagram of the plan view of the photonic crystal. Light propagates along the direction of the channel.

The proposed structure is a two dimensional square lattice PC designed on a substrate of RI 1.457334. The PC is constructed on a polymer base of RI 1.327 (MY-132-V15K) with nanorods (diameter $80 \mathrm{~nm}$ and pitch (a) $200 \mathrm{~nm}$ ) of SOPRA-polysilicon 2 with $10 \%$ silicon. The in-plane PBG of the PC is shown in Fig. 2.

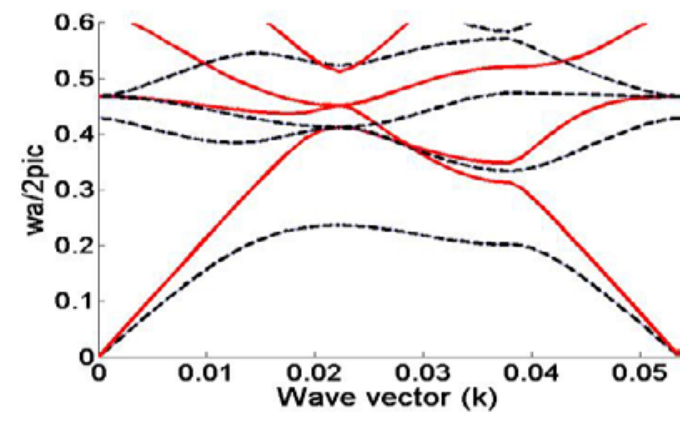

Fig. 2. Bandgap diagram of the PC. Dashed lines are for TM and solid lines are for TE polarization. $\omega$ is operating frequency, a is pitch, $\mathrm{c}$ is velocity of light in vacuum.

The structure has a TM band gap between 588nm to 833nm, but no TE band gap is present. The in-plane DOS (density of state) plot for the structure is given in Fig. 3. The DOS plot shows the variation of the number of permissible EM modes in the crystal structure with normalized frequency [3].

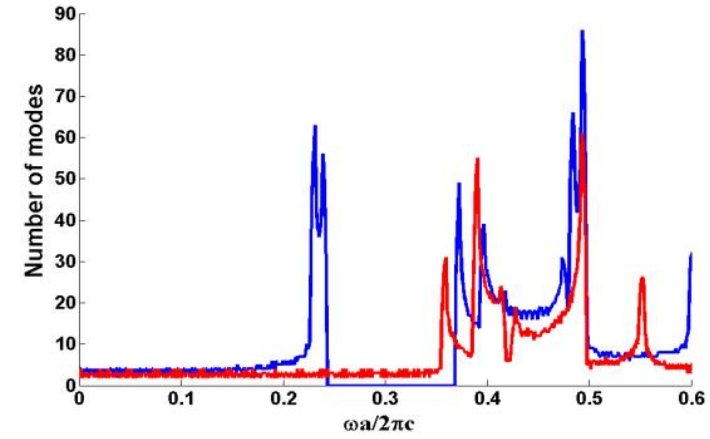

Fig. 3. The DOS plot of the proposed photonic crystal. The red line is for TE modes and blue line for TM modes.

It can be shown from Fig. 3 that there are no TM modes when $\omega \mathrm{a} / 2 \pi \mathrm{c}$ varies from 0.24 to 0.34 . On the other hand this region has nonzero DOS value for TE modes. This means in the mentioned frequency band only the TM mode will propagate through the defect channel.

\section{B. Design of plasmonic sensor}

We are interested to design an integrated sensor. We first took a four layer plasmonic waveguide structure. The schematic diagram of the plasmonic structure is shown in Fig. 4.

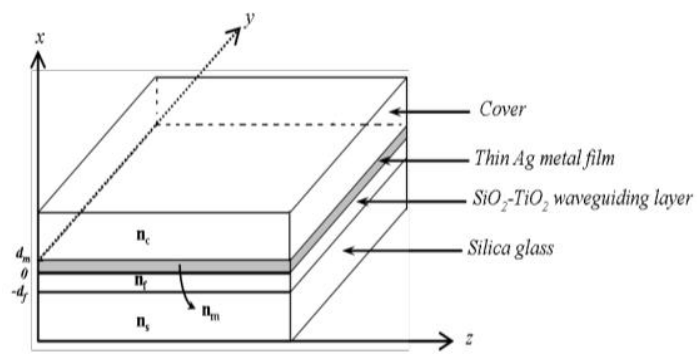

Fig. 4. . Four layer planar waveguide structure with embedded thin metal layer of silver (Ag).

The structure is assumed to be infinite in y direction and the refractive index distribution is given by [4].

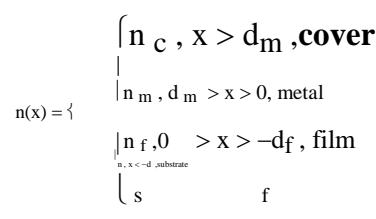

The structure is optimized to support only the fundamental modes in the specified bandgap region. The refractive index of the guiding layer is taken as 1.7264. In order to achieve a better coupling between the plasmonic mode and the guided mode we set the thickness of the metal layer to $40 \mathrm{~nm}$. 

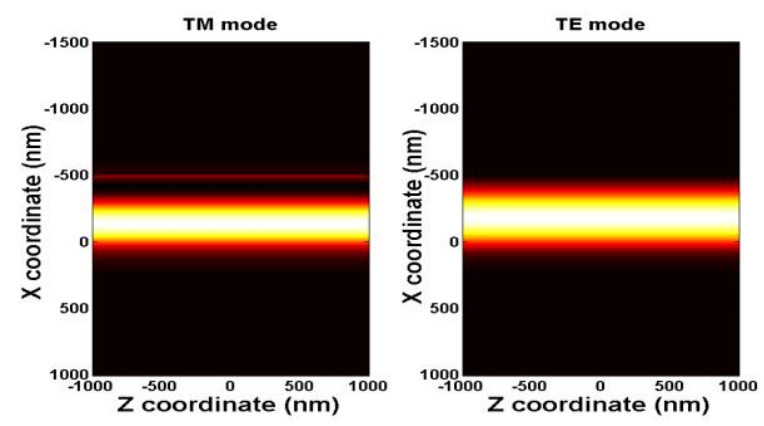

Fig. 5. Fundamental TM and TE mode field distribution for ns=1.457334, $\mathrm{nf}=1.7264, \mathrm{nc}=1.33, \mathrm{df}=550 \mathrm{~nm}$ and $\mathrm{dm}=40 \mathrm{~nm}$. The metal layer is considered as silver layer. RI of Ag is calculated from ref. [5].

Now we varied the thickness of the guiding layer and found out that when $\mathrm{df}$ is below $580 \mathrm{~nm}$ only the fundamental TE and TM modes are supported in the structure. This optimization is carried out by fixing the cover layer index to 1.33 and operating wavelength is taken $632 \mathrm{~nm}$. The modal field distribution is shown in Fig.5.

The interaction between the core guided fundamental TM mode and the surface plasmon polariton (SPP) can be enhanced farther by wall perturbation method. If the interface between the metal layer and the adjacent dielectric layer is perturbed in a symmetric fashion then the plasmonic response of the structure increases. The schematic diagram with triangular perturbation is shown in Fig. 6.

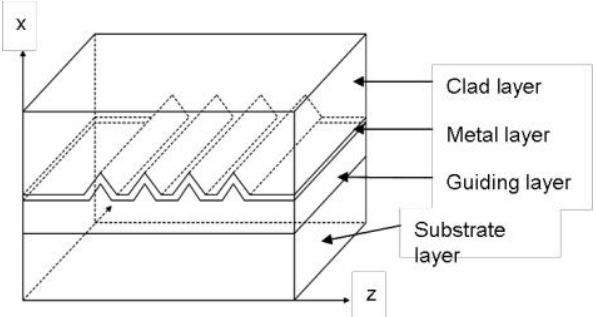

Fig. 6. . Schematic diagram of the waveguide with triangular wall perturbation with surface metal layer.

In an earlier study we showed that the wall perturbation increases the surface plasmon (SP) amplitude [4]. Hence more power will be coupled out from the guided mode. The variation of SP amplitude with different cover index for various perturbations is shown in Fig. 7.

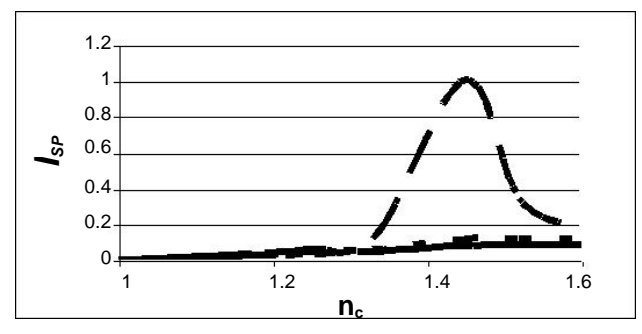

Fig. 7. Variation of normalized SP amplitude (ISP) at metal-clad interface with clad index (nc) for different grating geometry at the interface. Solid line for without perturbation, dashed line for rectangular and broken line for triangular perturbation.
We now integrated the two structures to design a polarization selective plasmonic sensor. The main advantage of this integrated structure is its efficiency. Since the guided mode in the PC is used to excite the plasmonic structure so better light coupling is expected. The schematic diagram of the integrated device is shown in Fig. 8.

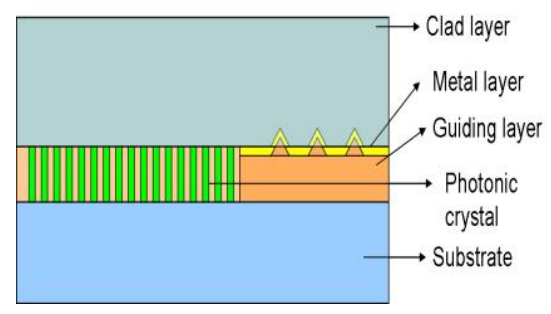

(a)

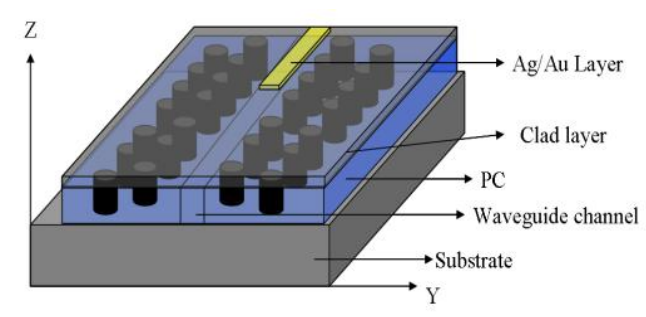

(b)

Fig. 8. (a) Cross-sectional schematic view of the integrated sensor device. (b) 3D schematic view of the sensor structure

\section{SiMULATION AND RESULTS}

We analyzed wave propagation through the structure y Lumerical FDTD engine to observe the evolution of the propagated core guided mode. We calculated the change in the guided mode amplitude for different refractive index of the clad layer. We set the wavelength to $632 \mathrm{~nm}$ as LED sourced at this wavelength is widely available. We set the cover layer index to 1.33 (water) as the intention is to use it as a biosensor. We excite the structure with a plane wave source. The propagation of fundamental TE and TM mode is shown in Fig. 9.

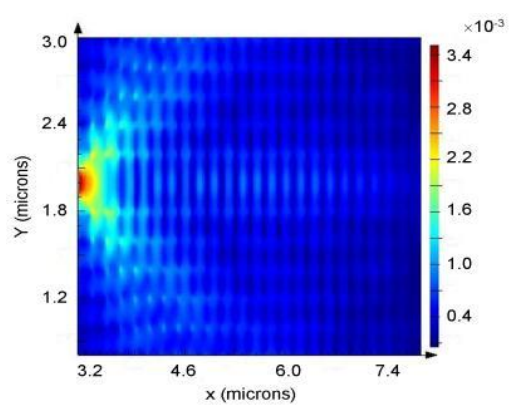

(a) 


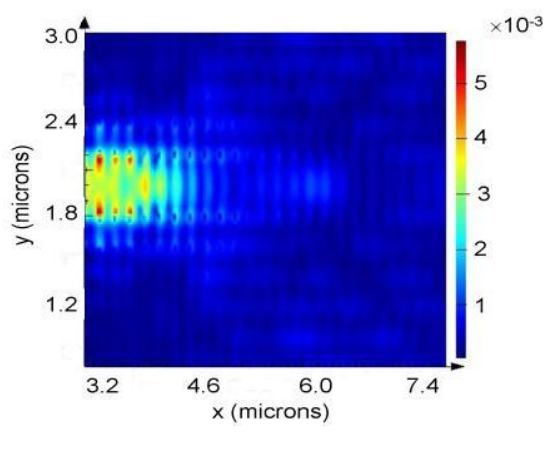

(b)

Fig. 9. Propagation of light through the integrated sensor (a) TE polarized (b) TM polarized

From Fig. 9 one can clearly infer that only the TM mode will be coupled to the sensor structure. The TE light readily leaks out through the PC cladding within a propagation distance of $1 \mu \mathrm{m}$. We took the cavity channel $5 \mu \mathrm{m}$ long and the sensor structure $(3 \mu \mathrm{m}$ long) is embedded within it. The normalized TE and TM mode amplitude after a propagation length of $8 \mu \mathrm{m}$ for different cover index is shown in Fig. 10.

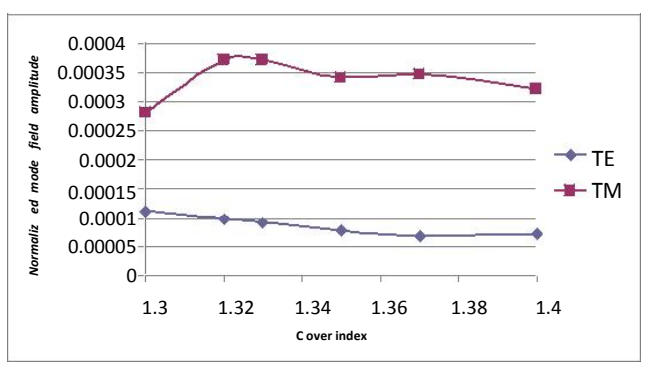

Fig. 10. Variation of normalized TE and TM output amplitude with cover refractive index.

We can conclude from Fig. 10 that TM polarized output dominates in the output signal. We compared the output TM and TE amplitude ratio of the sensor structure embedded in the PC and without PC. The result is shown in Fig. 11.

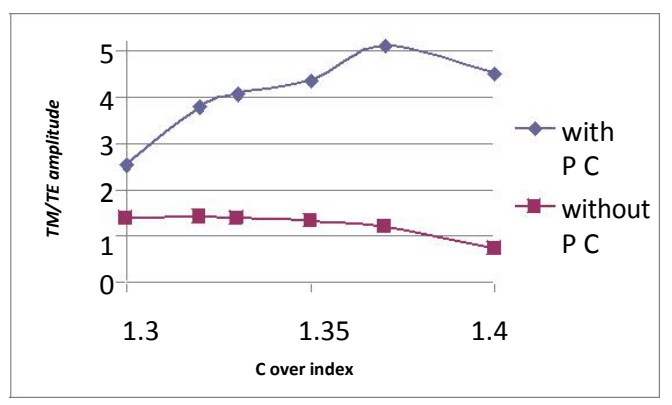

Fig. 11. TM/TE amplitude variation with cover refractive index TM/TE amplitude variation with cover refractive index.
Fig. 11 shows that detection of TM signal is much easier in our proposed structure. So the SNR will increase as the TE output will act as noise. The PC helps to cut down the TE response and thus the structure gives a better signal detection.

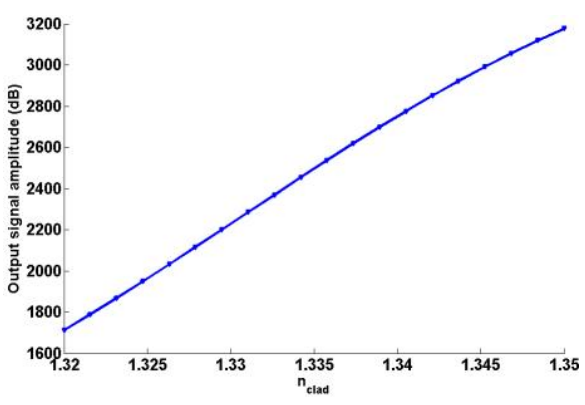

Fig. 12. . Variation of output signal TM amplitude with Cover refractive index.

Since we intend to use amplitude variation measurement technique to detect the sample, which will act as cover layer, it is needed that the output signal should vary linearly in the desired RI region. Fig. 12 shows that the signal varies linearly in the region 1.32 to 1.35 , which is the most desired range. The sensitivity is $50238 \mathrm{~dB} / \mathrm{RIU}$.

\section{CONCLUSION}

The proposed sensor offers linear and polarization selective operation in the RI region suitable for bio-fluids. The refractive index of the cover medium can be estimated by observing the amplitude of the output signal. The intensity of the output signal changes linearly with cover index. The introduction of PC enhances the SNR ratio and the perturbation increases the sensitivity. The total structure is compact and can be designed on a chip.

\section{Acknowledgement}

Director, CSIR-CGCRI and CSIR-12th plan project GLASSFIB.

\section{References}

[1] J. Homola, S. S. Yee and G. Gauglitz, "Surface plasmon resonance sensors: review”, Sensors and Actuators B, 54, 3-15 (1999).

[2] A. A. Mradudin and A.R. McGurn, "Out of plane wave propagation of electromagnetic waves in a two dimensional periodic dielectric medium”, J. Mod. Opt. 41, No-2, 275-284 (1994).

[3] K. Sakoda, Optical properties of photonic crystal (Springer-Verlag, Berlin Heidelberg, 2005).

[4] R. Chattopadhyay, R. D. Roy and S. K. Bhadra, "Sub-micron plasmonic waveguide for efficient sensing of bio-fluids”, PIER Lett. 41, 1-12 (2013).

[5] A. D. Rakic', A. B. Djuriš ic' , J. M. Elazar, and M. L. Majewski, "Optical properties of metallic films for vertical-cavity optoelectronic devices”, App Opt. 37, No. 22, 5271-5283 (1998). 